\title{
Clinical Applications of TD-NIRS: a Widespread Scenario
}

\author{
R. Re ${ }^{* 1,2}$, L. Spinelli ${ }^{2}$, D. Contini ${ }^{1}$, I. Pirovano ${ }^{1}$, M. Zanoletti ${ }^{1}$, R. Cubeddu ${ }^{1}$ and A. Torricelli ${ }^{1,2}$ \\ ${ }^{I}$ Dipartimento di Fisica, Politecnico di Milano, Piazza Leonardo da Vinci 32, 20133 Milan, Italy; ${ }^{2}$ Istituto di Fotonica e Nanotecnologie, \\ Consiglio Nazionale delle Ricerche, Piazza Leonardo da Vinci 32, 20133 Milan, Italy. \\ rebecca.re@polimi.it
}

\begin{abstract}
TD-NIRS allows the monitoring of tissue hemodynamics enhancing the contribution from the late (deeper) photons. After a brief review of its peculiarities, examples of its employment in clinics, on brain and muscles, will be presented (C) 2020 The Author(s)
\end{abstract}

\section{Time Domain Near Infrared Spectroscopy: TD-NIRS}

Near infrared spectroscopy (NIRS) is a spectroscopic optical technique which makes use of light in the 600-1100 nm window to assess the optical tissue properties, i.e. absorption and scattering coefficient. This technique is fast, noninvasive and non-destructive, therefore it can be widely used in real-time, in-vivo applications on human. The time domain (TD) approach [1], based on the acquisition of the photon distribution of time-of-flight (DTOF), add to the conventional NIRS techniques, i.e. continuous wave CW-NIRS, some more features that can be summarized as follow: 1) capability to estimate the absolute values of the optical properties since they are directly dependent on the changes of the DTOF shape; 2) the information on the photon time of arrival (i.e. the photon path length) is encoded in the DTOF. It is possible to exploit this characteristic to enhance the contribution of those photons coming from the deeper layers of the tissue investigated, avoiding the confounding effects of the more superficial ones; 3 ) acquisition with an higher contrast and depth resolution respect to $\mathrm{CW}$ are achievable; 4) fluctuations in the signal in terms of number of counts don't affect the detected signal, making negligible the effects of a non-perfect contact or of the moving artefacts; 5) possibility to extrapolate from the absolute values of the optical coefficients, the absolute values of the chromophores useful in clinical application. Example are, but are not limited to, oxyhemoglobin $\left(\mathrm{O}_{2} \mathrm{Hb}\right)$, deoxy-hemoglobin $(\mathrm{HHb})$, oxygen saturation $\left(\mathrm{SO}_{2}\right)$ and total haemoglobin $(\mathrm{tHb})$. These parameters allow the monitoring of the hemodynamic state of the resting brain in patient with respect to healthy subjects or to evaluate its activation in response to stimuli (neuro-vascular coupling) or again to monitor the oxidative metabolism status of a muscular compartment during rehabilitation exercises.

\section{TD-NIRS in clinics}

The possibility to have absolute values of the hemodynamic tissue's parameters and to follow their variation in time, makes TD-fNIRS a good candidate for clinical applications in addition to standard techniques such as magnetic resonant imaging (MRI), electroencephalography (EEG) or electromyography (EMG). At the moment the TD-NIRS instruments, present in our research group, have the advantage to be portable with the possibility to monitor a patient at the bedside. Some examples are the instruments describe in Re et al. 2013 and 2018 [2,3]. In the following subsections we will present some clinical applications performed with these TD-fNIRS medical devices on brain or muscle. All the studies received the approval of the Ethical Committee of the hospital, where they were carried out, and were conducted in compliance with the Declaration of Helsinki.

\subsection{Brain: Ischemic stroke patients}

The aim of this work was to assess differences in the basal values of the hemodynamic parameters in the brain region affected by an ischemic stroke event. At "I.R.C.C.S. San Raffaele Hospital" in Milan, data from 88 controls and 47 ischemic stroke patients with large vessel occlusions were acquired. The ischemic group was divided in TACS/PACS (Total or Partial Anterior Circulation Syndrome, N=36) and LACS (Lacunar Anterior Circulation Syndrome, $\mathrm{N}=5)$. The first group was then split in recanalized $(\mathrm{N}=18)$ and non-recanalized $(\mathrm{N}=18)$ patients. For all the subjects, six standard positions on the frontal, central and parietal brain area were investigated, plus the positions where the ischemic events took place together with the corresponding positions in the contra-lateral hemisphere. We repeated the measurements three times in the six standard positions for the control group, demonstrating that the measurements are well reproducible and reporting ranges of normality for $\mu_{\mathrm{a}}, \mu_{\mathrm{s}}^{\prime}, \mathrm{O}_{2} \mathrm{Hb}, \mathrm{HHb}, \mathrm{SO}_{2}$ and $\mathrm{tHb}$. We also shown that with the increasing of age ( $>55$ years), the hemodynamic parameters have a significance decreasing [4]. In the large cohort of patients, we found significant differences in 
the hemodynamic parameters respect to the control group and also between affected and non-affected hemispheres. In particular, the $\mathrm{SO}_{2}$ seems to be the best fiducial marker in this kind of pathologies [5].

\subsection{Brain: Glaucoma patients}

Glaucoma affects the eye, damaging the optic nerve due to a high intraocular pressure (IOP) and causing blindness. Recently, with a MRI study an involvement of the brain occipital area was shown [6]. In collaboration with "ASST FBF Sacco Hospital" in Milan, we performed a functional study on 118 subjects divided in controls (31), subjects which presented a glaucomatous damage to the eye (further divided in Open Angle Glaucoma: 19, IOP $>21 \mathrm{mmHg}$ and Normal Tension Glaucoma: 24, IOP $<21 \mathrm{mmHg}$ ), and subjects with intraocular pressure (IOP) $>21 \mathrm{mmHg}$ in the absence of clinical signs of glaucoma (20). 12 subjects presented differences in the diagnosis of the two eyes and 12 were not eligible for clinical or experimental reasons. To all the subjects a visual stimulus (reversing checkerboard at $10 \mathrm{~Hz}$ ) was delivered for five times when they were fixing a monitor with one eye at a time. The $\mathrm{O}_{2} \mathrm{Hb}$ and $\mathrm{HHb}$ time courses during the repetitions were calculated and fitted with a canonical response function [7], obtaining the maximum and minimum amplitudes respectively for $\mathrm{O}_{2} \mathrm{Hb}$ and $\mathrm{HHb}$. We observed that in the glaucoma group, with respect to the control one, the amplitudes of the hemodynamic response showed a different pattern, confirming the hypothesis that there is a cerebral component in patient with glaucoma.

\subsection{Muscle: rehabilitation evaluation}

Two applications for oxidative muscular metabolism assessment during rehabilitation will be here presented. The two studies are still in progress. The first one is in collaboration with "ASST Gaetano Pini Hospital" in Milan; the hemodynamic status of the quadriceps muscle of around 100 volunteers will be monitored after a leg surgery, before and after a 15 days of physiotherapy training on the healthy leg. The patients had to performed isometric repeated contraction for a period of 5 minutes. The second one, in collaboration with "I.R.C.C.S. Medea Associazione la nostra famiglia" in Bosisio Parini (LC), is focuses on children (6-16 age) with cerebral disease affecting the motor cortex. In this case 15 children will be monitored before and after a 1 month of standard rehabilitation training, while 15 before and after special session of rehabilitation with a robotic support which allows to perform active and passive ankle movements. In this case the muscles investigated are the gastrocnemius and the tibialis anterior muscle. In both the studies the TD-NIRS acquisition are combined with EMG sensors [8]. The principal aim is to find a hemodynamic indicator of the progresses of the rehabilitation and to assess the muscular fatigue.

\section{Future: novel instruments and applications}

TD-NIRS is a unique non-invasive tool that the medical doctor can use directly in clinics to assess the hemodynamic parameters of brain or muscles. In the near future other body compartment could be monitored or spectrally characterized with this technique, such as breast or thyroid. From an instrumental point of view, we have to expect a drastic decrease in size and costs which will make TD-NIRS portable and wearable allowing new applications in the field of athletes' performances assessment or personalized patient monitoring at home.

\section{Acknowledgements}

This work was partially supported by Regione Lombardia and Fondazione Cariplo in the framework of the project "EMPATIA@Lecco - EMpowerment del PAzienTe in cAsa" (Rif.2016-1428).

\section{References}

[1] A. Torricelli et al. "Time domain functional NIRS imaging for human brain mapping," Neuroimage 85, 28-50 (2014).

[2] R. Re et al. "Multi-channel medical device for time domain functional near infrared spectroscopy based on wavelength space multiplexing,"

Biomed. Opt. Express 4(10), 2231-2246 (2013).

[3] Re et al. "Time Domain Near Infrared Spectroscopy Device for Monitoring Muscle Oxidative Metabolism: Custom Probe and In Vivo Applications," Sensors 18, 264-277 (2018).

[4] G. Giacalone et al. "Cerebral time domain-NIRS: Reproducibility analysis, optical properties, hemoglobin species and tissue oxygen saturation in a cohort of adult subjects," Biomed. Opt. Express 8 (11), 4987-5000 (2017).

[5] G. Giacalone et al. "Time-domain near-infrared spectroscopy in acute ischemic stroke patients," Neurophotonics 6(1), 1-10 (2019).

[6] N. Gupta et al. "Glaucoma as a neurodegenerative disease," Curr. Opin. Ophthalmol. 18(2), 110-114 (2007).

[7] Uga et al., "Optimizing the general linear model for functional near-infrared spectroscopy: an adaptive hemodynamic response function approach," Neurophotonics 1(1), 1-10 (2014).

[8] A. Scano et al. " NIRS-EMG for Clinical Applications: A Systematic Review," Appl. Sci. 9, 2952-2969 (2019). 